\title{
Retraction
}

\section{Retracted: Complexity Assessment of Chronic Pain in Elderly Knee Osteoarthritis Based on Neuroimaging Recognition Techniques}

\author{
Computational and Mathematical Methods in Medicine
}

Received 10 November 2022; Accepted 10 November 2022; Published 13 December 2022

Copyright (C) 2022 Computational and Mathematical Methods in Medicine. This is an open access article distributed under the Creative Commons Attribution License, which permits unrestricted use, distribution, and reproduction in any medium, provided the original work is properly cited.

Computational and Mathematical Methods in Medicine has retracted the article titled "Complexity Assessment of Chronic Pain in Elderly Knee Osteoarthritis Based on Neuroimaging Recognition Techniques" [1] due to concerns that the peer review process has been compromised.

Following an investigation conducted by the Hindawi Research Integrity team [2], significant concerns were identified with the peer reviewers assigned to this article; the investigation has concluded that the peer review process was compromised. We therefore can no longer trust the peer review process and the article is being retracted with the agreement of the Chief Editor.

\section{References}

[1] X. Wu, J. Liu, M. Liu, and T. Wu, "Complexity Assessment of Chronic Pain in Elderly Knee Osteoarthritis Based on Neuroimaging Recognition Techniques," Computational and Mathematical Methods in Medicine, vol. 2021, Article ID 7344102, 11 pages, 2021.

[2] L. Ferguson, "Advancing Research Integrity Collaboratively and with Vigour," 2022, https://www.hindawi.com/post/advancingresearch-integrity-collaboratively-and-vigour/. 


\title{
Complexity Assessment of Chronic Pain in Elderly Knee Osteoarthritis Based on Neuroimaging Recognition Techniques
}

\author{
Xuemin Wu ${ }^{D},{ }^{1}$ Jingjing Liu, ${ }^{1}$ Min Liu, ${ }^{2}$ and Tao $W u^{2}$ \\ ${ }^{1}$ Community Health Station, China University of Mining and Technology-Beijing, Beijing 100083, China \\ ${ }^{2}$ Beijing Institute of Geriatrics, Xuanwu Hospital, Capital Medical University, Beijing 100053, China
}

Correspondence should be addressed to Xuemin Wu; 201372@cumtb.edu.cn

Received 18 September 2021; Revised 6 October 2021; Accepted 30 October 2021; Published 28 November 2021

Academic Editor: Osamah Ibrahim Khalaf

Copyright (C) 2021 Xuemin Wu et al. This is an open access article distributed under the Creative Commons Attribution License, which permits unrestricted use, distribution, and reproduction in any medium, provided the original work is properly cited.

\begin{abstract}
The chronic pain of knee osteoarthritis in the elderly is investigated in detail in this paper, as well as the complexity of chronic pain utilising neuroimaging recognition techniques. Chronic pain in knee osteoarthritis (KOA) has a major effect on patients' quality of life and functional activities; therefore, understanding the causes of KOA pain and the analgesic advantages of different therapies is important. In recent years, neuroimaging techniques have become increasingly important in basic and clinical pain research. Thanks to the application and development of neuroimaging techniques in the study of chronic pain in KOA, researchers have found that chronic pain in KOA contains both injury-receptive and neuropathic pain components. The neuropathic pain mechanism that causes KOA pain is complicated, and it may be produced by peripheral or central sensitization, but it has not gotten enough attention in clinical practice, and there is no agreement on how to treat combination neuropathic pain KOA. As a result, using neuroimaging techniques such as magnetic resonance imaging (MRI), electroencephalography (EEG), magnetoencephalography (MEG), and near-infrared spectroscopy (NIRS), this review examines the changes in brain pathophysiology-related regions caused by KOA pain, compares the latest results in pain assessment and prediction, and clarifies the central brain analgesic mechanistic. The capsule network model is introduced in this paper from the perspective of deep learning network structure to construct an information-complete and reversible image low-level feature bridge using isotropic representation, predict the corresponding capsule features from MRI voxel responses, and then, complete the accurate reconstruction of simple images using inverse transformation. The proposed model improves the structural similarity index by about $10 \%$, improves the reconstruction performance of low-level feature content in simple images by about $10 \%$, and achieves feature interpretation and analysis of low-level visual cortical fMRI voxels by visualising capsule features, according to the experimental results.
\end{abstract}

\section{Introduction}

Approximately $80 \%$ of the information that humans obtain from the outside world comes from vision; therefore, vision plays an irreplaceable and crucial role in the process of knowing, understanding, and transforming the external world. The eye, retina, optic nerve, lateral geniculate body, and visual cortex of the brain make up the human visual system, which is an essential component of the nervous system. The eye projects visible picture information onto the retina, which is translated into electrical impulses and transferred from the optic nerve to the brain's visual cortex through the lateral geniculate body [1]. The visual cortex is the core of the human visual system and is mainly responsible for the advanced processing of visual information; through the hierarchical processing and processing of visual information, humans can understand the visual scene seen [2]. The processing and management of visual information in the visual cortex, which is made up of numerous nerve cells, is based on very complicated neural activity. Exploring the information processing mechanism of visual scene content in the visual cortex of the brain, analysing the characteristics of the representation of visual scene content by the neural activity of the visual cortex, and parsing the visual scene content in the neural activity of the visual cortex have been a hotspot in brain science, and it is a very important topic in 
the study of human visual function. In the absence of a full cure for KOA, nonpharmacological and pharmacological therapy focuses on alleviating joint discomfort and maintaining or enhancing joint function, with partial/total joint replacement surgery done only if normal joint function cannot be maintained by these techniques [3]. The lack of largescale, standardised, cross-population epidemiologic surveys of bone and joint health, as well as the social perception of osteoarthritis as a "normal part of ageing," the lack of medication durability and the high prevalence of complications, and the lack of large-scale, standardised, cross-population epidemiologic surveys of bone and joint health all pose challenges [4]. The absence of a large-scale, systematic, crosspopulation epidemiological study of bone and joint health has posed a number of difficulties to disease's treatment. As a result, it is critical to address the disease burden issues based on patient requirements, so that more patients may benefit from standardised therapy, enhance bone and joint health, and improve their quality of life.

In the field of sports biomechanics, mechanical loading plays a crucial role in the development of KOA. Due to the high degree of difficulty and narrow application of direct in vivo measurement of knee joint loading, the knee joint internal retraction moment obtained from gait analysis tests performed by a motion capture system in conjunction with a force table system can be used as a valid and reliable golden proxy for dynamic loading of the intraknee, femorotibial intercompartmental compartment. In simple terms, during the gait support period, an external moment that causes the knee joint to invert, squeezing the medial femorotibial interval and separating the lateral interval, is generated as the ground reaction force vector is biased toward the medial compartment of the knee joint quality of life and functional activity in KOA patients, with $21.1 \%$ to $66.7 \%$ of KUA out of abnormal pain sensitivity or pain suppression in older adults worldwide. Neuroimaging techniques play an important role in identification [5]. The processing and management of visual information in the visual cortex, which is made up of numerous nerve cells, are based on very complicated neural activity. Exploring the information processing mechanism of visual scene content in the visual cortex of the brain, analysing the characteristics of the representation of visual scene content by the neural activity of the visual cortex, and parsing the visual scene content in the neural activity of the visual cortex have been a hotspot in brain science, and it is a very important topic in the study of human visual function. In the absence of a full cure for KOA, nonpharmacological and pharmacological therapy focuses on alleviating joint discomfort and maintaining or enhancing joint function, with partial/total joint replacement surgery done only if normal joint function cannot be maintained by these techniques [3]. The lack of large-scale, standardised, crosspopulation epidemiologic surveys of bone and joint health, as well as the social perception of osteoarthritis as a "normal part of ageing," the lack of medication durability and the high prevalence of complications, and the lack of large-scale, standardised, cross-population epidemiologic surveys of bone and joint health all pose challenges [4]. The absence of a large-scale, systematic, cross-population epidemiological study of bone and joint health has posed a number of difficulties to disease's treatment. As a result, it is critical to address the disease burden issues based on patient requirements, so that more patients may benefit from standardised therapy, enhance bone and joint health, and improve their quality of life. The visual cortex is an important part of the cerebral cortex. Visual information reaches the visual cortex through the human eye via the lateral geniculate body, where it is continuously processed and processed to form visual perception. The visual cortex plays an extremely critical role in the formation of vision. Neurons/nerve cells are the basic information processing units of the visual cortex. Nerve cells are mainly composed of cell bodies, dendrites, axons, and synapses, and the cell bodies and dendrites of visual neurons are concentrated in the gray matter to form the visual cortex. Many neurons form a complex neural circuit between them, which can realize complex visual information processing structures and functions, thus forming a complex visual system. The brain is divided into two hemispheres, the left hemisphere visual cortex receives information entering the right visual field and the right hemisphere visual cortex receives information entering the left visual field. The visual cortex of the brain is mainly situated in the occipital lobe, and the visual cortex is divided into regions according to the Brodmann subdivision of the brain. Visual information via the lateral geniculate body is first transmitted to the primary visual areas of the human visual cortex, and then, the output information from the primary visual areas is transmitted to the higher visual areas layer by layer through two pathways. Visual information in the visual cortex is continuously processed and processed hierarchically, i.e., visual information flows continuously from the lower visual areas to the higher visual areas, and the lower visual information features are gradually transformed into higher visual information features.

\section{Current Status of Research}

Degenerative lesions of articular cartilage, secondary osteophytes, and aseptic inflammatory lesions in KOA patients are the main causes of chronic pain, swelling, and stiffness in the knee joint U7I, and the symptoms gradually worsen as the disease progresses, and KOA patients often have limited joint function, which can lead to disability in severe cases [6]. Leroux et al. conducted a survey of 110 patients with osteoarthritis in a community in Shanghai and found that $27.4 \%$ of KOA patients had depressive symptoms [7]. A study by Vadivelu et al. found that $47.4 \%$ of KOA patients had anxiety or depression or both, and both anxiety and depression scores were higher than local normative levels [8]. 38.5\% of older adults with osteoarthritis had depressive symptoms, as reported by Tracey et al. predictors of depression in patients with arthritis [8]. Depression and anxiety in patients with KOA are not encouraging. However, depression and anxiety symptoms often go unnoticed by health care providers, directly contributing to further decreases in inpatient QOL [9]. KOA fatigue symptoms are not routinely assessed in clinical evaluations, and patients often do not actively respond to health care providers about their fatigue. 
A recent study found that of 231 patients with knee or hip osteoarthritis, $47 \%$ of them had severe fatigue [10]. Fatigue can lead to reduced physical activity in KOA patients, which directly affects their motivation to participate in social activities and can negatively affect their QOL. Ravat et al. investigated the sleep status of 2682 patients with osteoarthritis and found that the prevalence of sleep disorders was approximately $71 \%$, with insomnia being the most common sleep disorder in KOA patients [11].

The internal changes in the knee joint that result from the disease include cartilage destruction, subchondral bone thickening, and new bone reconstruction. These alterations result in discomfort, instability, stiffness, and edoema in the knee, necessitating arthroplasty [12]. The pain in the knee caused by OA is usually bilateral, occurring in and around the knee joint and spreading to the groin and anterior or lateral thighs. The symptoms of $\mathrm{OA}$ include decreased joint mobility, joint swelling/synovitis (fever, effusion, and synovial thickening), twisting, periarticular pressure, bone swelling, and deformity due to bone growth. OA patients have decreased ability to perform basic daily activities (e.g., climbing stairs, changing from a sitting to a standing position). The main symptoms of osteoarthritis of the knee are pain, stiffness, reduced range of motion, twisting pain, and swelling [13]. Pain in the early course of the disease is usually described as a dull intermittent pain, confined to one chamber. It usually worsens with increased activity and relieves with rest [14]. As the disease process worsens, all chambers of the femorotibial joint are involved, and joint pain becomes more constant and diffuse, with pain occurring at rest and night. The exact source of joint pain due to KOA is not known, and Dieppe and Lohmander suggest that it is essentially due to biological, psychological, and social factors [15]. Prolonged sitting and stair climbing pain suggest femoral pin joint involvement, and the above may be associated with mechanical symptoms, meniscal abnormalities, sparing osteochondral fragments, or significant joint surface abnormalities.

Pain, especially chronic pain, is a major symptom of KOA, and its effects on physical disability, motor function, and negative mood can significantly reduce patients' quality of life and can even lead to cognitive impairments such as poor decisionmaking and abnormalities in the body's sensory, emotional, and cognitive brain activity. Kellgren and Lawrence imaging categorization, illness duration, and body mass index are all variables that influence the physical condition of KOA patients, according to recent research (BMI), while the factors affecting the psychological status are mainly the disease duration. Although degenerative changes in the knee are the initial trigger for chronic pain in $\mathrm{KOA}$, there is an inconsistency between local imaging of the knee and patient's pain. In recent studies, factors such as central sensitization and neuroplasticity are coming into focus. The transformation of KOA pain from acute to chronic pain causes complex pathophysiological changes in the brain, and both structural and functional brain alterations may be present in chronic moderate and severe pain. The oil spill detection results of two oil spill identification models that consider environmental and characteristic factors are fused at the decision level using D-S evidence theory improved by fuzzy set theory, and the oil spill detection effects of the above two oil spill detection methods are analysed and compared to see how effective the decision analysis is.

\section{Analysis of Neuroimaging Recognition Techniques for Assessing the Complexity of Chronic Pain in Elderly Knee Osteoarthritis}

3.1. Design of Neuroimaging Recognition Techniques for Osteoarthritis of the Knee in the Elderly. When a natural picture stimuli is given to a person, the visual cortex of the brain is engaged in response (as illustrated in Figure 1). The response values of the voxels of interest distributed in the three-dimensional space of the visual cortex to the natural image stimulus can finally be obtained as a measure of the neural activity response in the corresponding region of the voxel by using fMRI to record the BOLD signal associated with the neural activity of the visual cortex [16]. The response values of many voxels in the cortex form the voxel response vector. Therefore, in this way, a series of visual cortex voxel response vectors can be obtained by continuously presenting images from the natural image database to the subjects, and they correspond to the images one by one so that the fMRI dataset corresponding to the natural image stimulus dataset can be constructed as the database for subsequent visual information parsing.

The image datasets used as visual stimuli are usually selected from publicly available natural image databases, and for subsequent training and validation of the computational model, the datasets are usually divided into training and test sets. The stimuli are then played back to the subjects for viewing based on predetermined presentation times, presentation frequencies, rest breaks, etc., usually in a randomly selected order using stimulus presentation software such as E-Prime. Usually, a single image presentation and data acquisition are referred to as a trial (trial), such as alternating presentation using a $200 \mathrm{~ms}$ frequency and a gray background for $I$ seconds, followed by the presentation of a gray background for 3 seconds for a total of 4 seconds, followed by a trial of presentation of the next image stimulus. The constant presentation of several image stimuli is usually performed as a single scan (run) and multiple scans from a single experiment (session), and usually, the presentation of the entire database image is divided into several experiments. In addition, the images as a test set are usually repeatedly presented several times for improving the signal-to-noise ratio of the BOLD signal [17]. During subjects' viewing of the images, the raw fMRI data of the subjects were collected, and then, the raw data were subjected to preprocessing operations such as $3 \mathrm{D}$ reconstruction, alignment, and head movement correction using software such as statistical parametric mapping (SPM) to obtain the BOLD responses of the voxels in the corresponding visual areas of interest over time when the subjects viewed different image stimuli temporal signal, reflecting the neural activity of the brain in response to the corresponding image stimuli. The amplitude of the BOLD response signal is usually obtained by deconvolution as the response value of the visual cortical voxels in the brain 


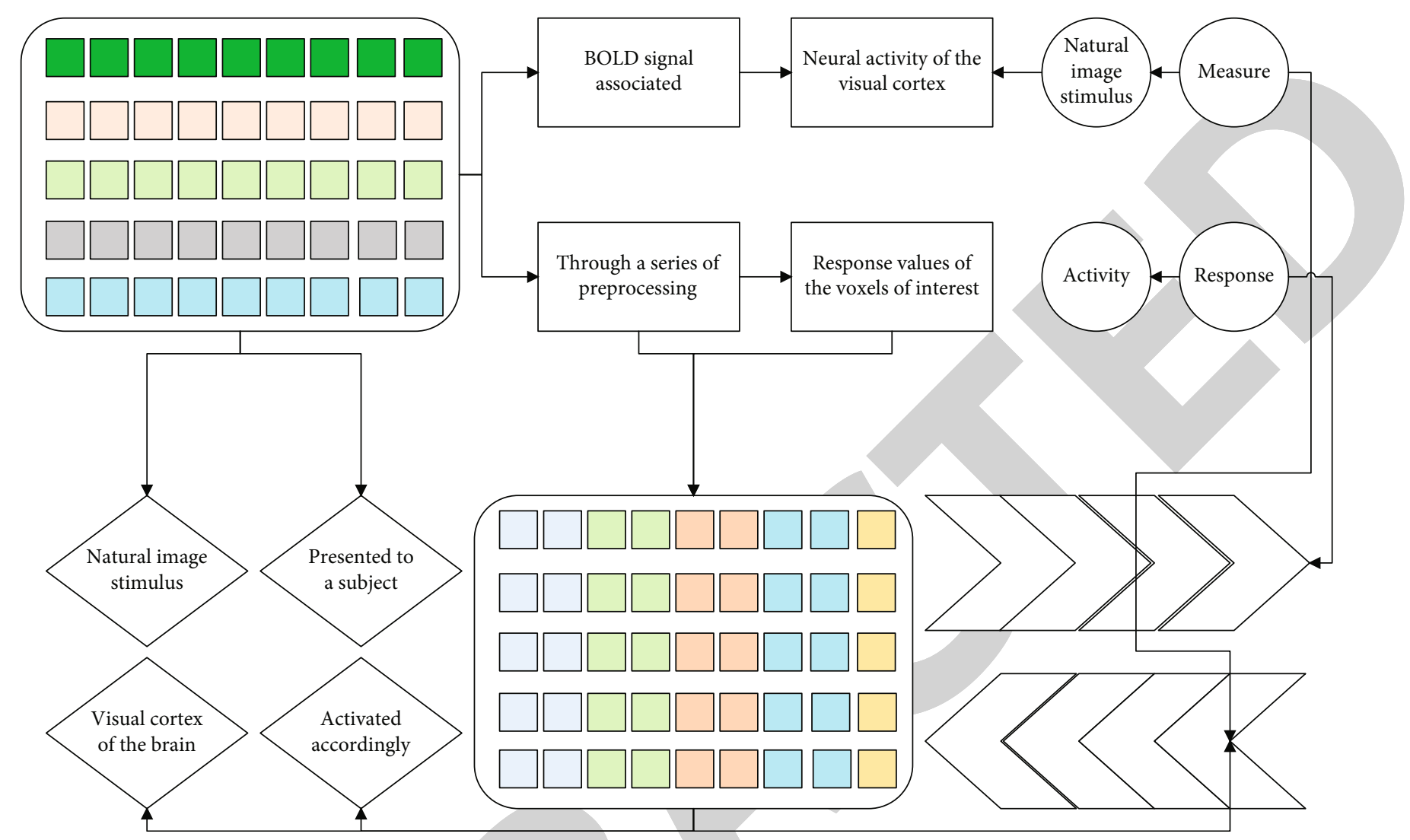

FIGURE 1: Visual stimulation experiments and database construction.

corresponding to the image stimulus. The neural response of the visual cortex of the brain to one picture stimulus is made up of the response values of thousands of voxels relating to that one image stimulus. In this way, a vector of cortical voxel responses corresponding to each image stimulus can be obtained, which makes up the final fMRI dataset. In addition, multiple subjects are usually selected to perform the same experiment and to acquire and process the data, thus including multiple sets of fMRI data from multiple subjects for subsequent validation of the robustness of the computational model on different subject data [18].

$$
\begin{aligned}
& f_{i}=f_{w_{c}}\left(S_{i}^{2}\right), \\
& v^{\prime}=w_{f c} f_{i}-b .
\end{aligned}
$$

This study was conducted by uniformly trained investigators to collect the questionnaire data and to ensure a generally consistent understanding of each entry in the scale. First, consent was obtained from the director of the hospital nursing department, and support was obtained from the head nurse of the department. Subsequently, study participants who met the inclusion criteria were screened through hospital's electronic medical record system. Consent was obtained from the patients following the voluntary principle. Patients and their families were informed about study's aim in order to earn their confidence and participation. The requirements and precautions for completing the questionnaire were explained in detail to the patients who voluntarily completed it independently.

$$
\begin{gathered}
S_{\text {shoot }}(t)=\operatorname{Re}\left[\operatorname{rect}\left(\frac{t}{D T}\right) e^{i \pi g_{r} t^{2}} e^{2 \pi w_{c} t}\right], \\
\operatorname{cor}_{k}=\frac{\operatorname{Cov}\left(v_{k}, v_{k}^{\prime}\right)}{\sqrt{\operatorname{Var}\left(v_{k}^{2}\right) \operatorname{Var}\left(v_{k}^{\prime}\right)}} .
\end{gathered}
$$

Rather of building the loss function by averaging the anticipated relevance values across all voxels in the visual ROI, a weighted relevance loss function is introduced, allowing the model to concentrate more on those voxels that are valid during optimization [19]. Also, noise regularization is added to the loss function to prevent the predicted voxels from continually getting larger, rendering the added Gaussian noise ineffective. The magnitude of muscle strength is important for maintaining joint stability. One of the most dominant large muscle groups around the knee joint is the quadriceps, and during a person's walk, rapid knee flexion occurs now the heel touches the ground, at which point the knee flexion is accomplished by centrifugal contraction of the quadriceps. The main role of the quadriceps during this phase of action is to prevent excessive knee flexion angle or too rapid flexion. The results of related studies confirm that groups with osteoarthritis of the knee usually exhibit inadequate knee extensor muscle strength compared to matched healthy populations. However, the role of knee 
extensor muscle weakness as a risk factor for the development of knee osteoarthritis is not fully understood. The knee extensor muscles may serve to reduce joint shock and stabilize the joint, thereby protecting the joint surface during weight-bearing and exercise. Related studies have demonstrated that greater mechanical loading of the articular cartilage caused by muscle weakness can cause degenerative changes in the joint. However, it remains to be proven whether knee extensor strength training can reduce the occurrence and development of knee osteoarthritis.

$$
L=\frac{\sum_{k} n_{k} \operatorname{cor}_{k}}{n}-\gamma\left|\frac{\sum_{k} n_{k} \operatorname{cor}_{k}}{n}\right| .
$$

Currently, there are differences in objectified diagnostic methods, differentiation criteria, and interpretation of results for the KOA phenotype. Neuroimaging techniques help in the diagnosis of pain types in KOA patients and provide a basis for differentiating or optimizing clinical treatment options. No single functional imaging method has superior temporal and spatial resolution at the same time. Task-related or resting-state functional magnetic resonance imaging (fMRI or rsfMRI) primarily quantifies brain functional indicators, such as FC, ReHo, and ALFF, and assesses brain functional changes through hemodynamic effects, with high temporal resolution; voxel-based morphometry (VBM) primarily quantifies anatomical brain structural indicators, such as gray matter density or volume, with high spatial resolution. With the application of neuroimaging techniques, the exact physiological significance represented by functional and structural changes in KOA chronic pain is more clearly defined. Arterial spin labeling methods can independently assess baseline physiological status; multimodal imaging, combining fMRI, EEG, and MEG, facilitates the determination of the order in which brain regions respond to painful stimuli. fMRI was combined with positron emission tomography by Fox et al. to analyze patterns of activation and inhibition of brain activity, a method that has now been applied to reveal the neurophysiology of meditative practices, as shown in Figure 2.

In studies of analgesic effects, fMRI can provide more objective evidence in revealing neuropathic pain mechanisms, can detect neural activity in injurious and painprocessing areas of the brain, can assess FC between brain regions such as the ACC and brainstem, can sensitively and objectively evaluate KOA skeletal femoral articular cartilage pain pressure based on the degree of PAG activation, and can also be used to characterize CNS activity in chronic pain. Each KOA phenotype contains many typical pain or pathophysiological mechanisms, and neuroimaging can be applied to detect pain parameters in patients.

It is well known that mechanical factors play a decisive role in the occurrence and development of osteoarthritis to some extent; therefore, it is particularly important to further understand and investigate the mechanical indicators related to the joints of the lower limbs of patients with osteoarthritis during their daily physical activities, with a view to better understanding the mechanical pathogenesis of this disease

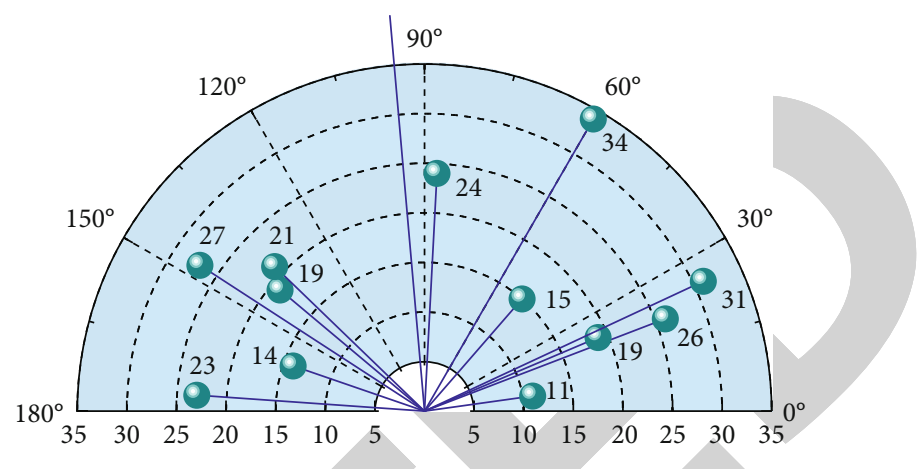

Figure 2: Visual coding effect.

and providing theoretical support for the treatment and prevention of osteoarthritis. Among the existing dynamic assessment tests of sports biomechanics, gait analysis of this group shows the advantages of this specialized field, which uses sports biomechanics theory, the processing means, and known human anatomy and physiology concepts to analyze the spatio-temporal parameters of human gait during walking, and a comprehensive gait analysis system consisting of a three-dimensional motion capture system, a force measurement table system, etc., which enables it to be used in sports rehabilitation. It is gradually recognized and accepted in the field of scientific research. The composite system can provide the spatial displacement, velocity, acceleration, ground reaction force, joint kinematics, and kinetics of the human body during walking without external interference.

3.2. Experimental Design for Assessing the Complexity of Chronic Pain in Elderly Knee Osteoarthritis. The study subjects were chosen strictly according to the inclusion and exclusion criteria; the appropriate survey time (after meals, avoiding patients' treatment or examination time) and survey location were chosen; simple explanations were chosen to encourage patients to participate; there were many questionnaire entries, and patients could rest if they became tired [20]. The investigator will make rounds on time during the process of patients filling out the questionnaire, and if patients have questions about an option, they can directly consult with the researcher; after the questionnaire was completed, it was collected in time to check the quality of the completed questionnaire to avoid omissions. To guarantee data's reliability, outliers and missing values are detected and handled. To prevent affecting the test findings and generating incorrect conclusions, verify that the data meets the test method's relevant criteria before settling on a statistical test technique. In the human visual system, visual information flows from the lower visual cortex to the higher visual cortex, and this hierarchical information processing enables people to understand the external world as they see it. Similarly, deep network models perform a specific visual task through a layer-by-layer representation of the input image. Figure 3 shows the encoding performance of the deep network image description model from low-level to high-level features on the low-level and high-level visual cortex, 


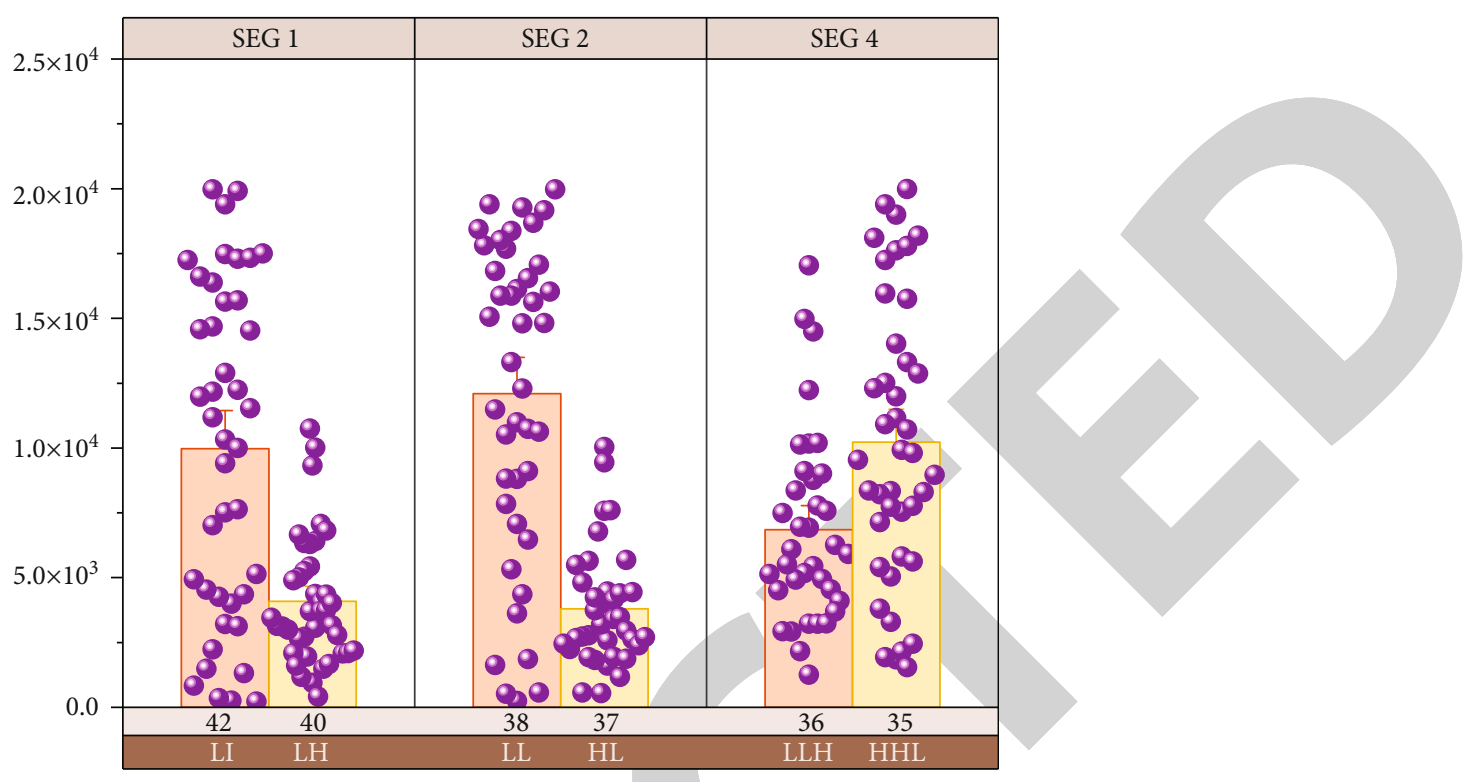

FIGURE 3: Hierarchical coding performance.

respectively, and the encoding performance is assessed by calculating the average correlation of all voxels.

Although the erosion of the disease occurs within the knee joint, internal changes over time inevitably cause changes in external joint movement and physical activity status. Concerning gait-motor characteristics, numerous studies have previously shown that older adults with knee osteoarthritis walk slower and with reduced stride length compared to healthy controls. Researchers generally believe that the involvement of KOA produces some structural and functional changes within the knee joint, such as increased joint pain, swelling and stiffness, reduced joint muscle strength, and limited range of motion. The researchers in this study requested the participants to perform the test at a comfortable and natural pace, not only to guarantee that subjects' true walking circumstances and characteristics were collected but also to further evaluate the effect of the illness on subjects' walking process. The pace results were consistent with the study hypothesis, and the data results showed that older women with knee osteoarthritis were significantly slower than healthy older women, a result that is consistent with many previous studies. Confirming this performance as an adaptive change prevalent in the KOA population, it has been theorized that the slower gait speed may be a strategy to reduce joint pain and/or decrease the load on the affected joint. Reduced walking speed has been linked to joint space narrowing, higher inflammatory mediator concentrations, discomfort, and quadriceps muscle weakness in people with knee osteoarthritis, according to studies. As you walk faster, your heart rate rises. As walking speed increases, intra-articular stress increases. In animal models, repeated high-intensity dynamic loading of joints has been shown to lead to chondrocyte death and the development of osteoarthritis. Increased walking speed combined with abnormal joint biomechanics may further amplify dynamic joint loading and accelerate joint degeneration. Reduced walking speed is thought to be a mechanism used by individuals with knee disease to reduce loading and pain in the medial compartment of the knee (as shown in Figure 4).

Increased foot advance angle is more common in the KOA population and may be an adaptive change to abnormal medial knee loading, and our findings confirm this inference, with transient values of foot advance angle shifting toward the outer eight in older women with KOA at the end of gait support. The results of a small number of international studies have shown that increasing the lateral foot angle is associated with a reduction in knee loading during walking on horizontal surfaces, running, or stair climbing in both healthy and KOA groups. In KOA individuals, self-selected lateral foot gait has a similar effect, as increasing the lateral foot angle beyond the original self-selected angle size reduces knee frontal plane internal loading and femoral tibial internal loading. However, there is a critical point at which such an increase would be impractical in practice, such as 90 degrees, so in future programmes where changing the foot advancement angle is the control focus of the gait modification programme, the KOA individual's current foot advancement angle and foot rotation ability should be taken into account and adjusted accordingly after a comprehensive assessment.

However, although increasing the lateral foot angle reduces knee stress towards the conclusion of the brace, it may have a negative impact at the beginning of the brace. Although not all evidence supports this claim, a limited number of studies have shown that increasing the lateral foot angle reduces end-of-support knee loading while increasing intrafrontal knee loading at the start of support [21]. Initial knee loads of support are usually higher than end-ofsupport knee loads in the KOA population, and clinical rehabilitation focuses on the first peak value of the internal knee moment. As a result, enhanced foot rotation with increased external octagonal angle may be a gait intervention for people with higher knee loading towards the end of gait 


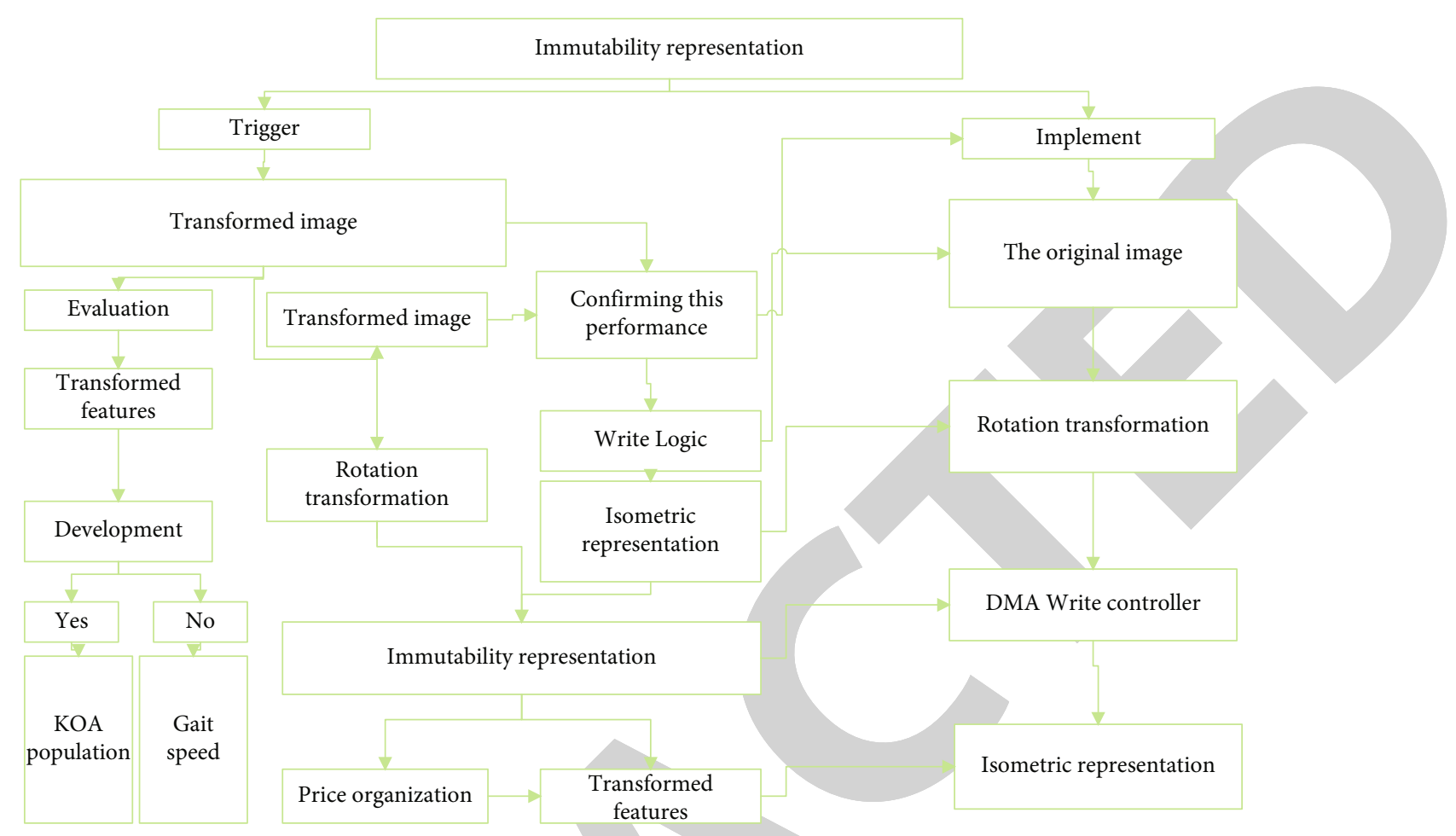

FIGURE 4: Difference between image invariance and isotropic characterization.

support, but its impact on knee loading at the start of support should be carefully monitored. In the middle and late stages of gait support, there was a greater group difference in the foot link advancement angle on the ipsilateral side of the diseased knee in older women with KOA compared to healthy older women; throughout the gait support period, the pelvis became less tilted in the frontal plane, the left-right angle became asymmetrical and more biased toward the affected side, the tilt remained asymmetrical and more biased toward the affected side, the tilt remained asymmetrical, and the phase of the double-peak appearance of the internal contraction moment matched. Therefore, these indicators may be more sensitive to changes in the double peak of the knee inversion moment.

\section{Analysis of Results}

4.1. Results of Neuroimaging Recognition Techniques for Osteoarthritis of the Knee in the Elderly. The reversible capsule features corresponding to the voxel responses can be predicted by a 3-layer neural network, so that the numerical relationship between the output (predicted reversible capsule features) and the input (voxel responses) is obtained using backpropagation through the gradients of the threelayer neural network. Thus, for specific dimensions in the capsule feature vector, it is possible to obtain which voxels are more active than others and to further obtain a characteristic interpretation of the voxels based on the relative values of the gradients. Figure 5 presents the relationship between voxels and specific interpretable features, from which the characterization of different voxels for attributes such as target shape and orientation in the image can be obtained, e.g., voxel 6 is sensitive to circle size and line thickness, while voxel 13 is more sensitive to orientation.

The current CNN feature-based visual reconstruction model first decodes the corresponding image features from the brain fMRI voxel response as an intermediate bridge and then reconstructs the image stimuli from the decoded intermediate features. Considering that reversible capsule features are characterized by feature reversibility and integrity, they can better characterize the information representation of the low-level visual cortex. Therefore, this chapter proposes a visual reconstruction model based on the capsule network structure from the perspective of deep learning network structure, which improves the accuracy level of simple image stimulus reconstruction by considering isotropic representation features. The model is first trained in an end-to-end manner to obtain a nonlinear mapping from images to reversible capsule features, and a nonlinear mapping from reversible capsule features to images. Then, the nonlinear mapping from the fMRI data of selected voxels to the reversible capsule features is trained. Finally, the trained image reconstruction module reconstructs the image stimuli using the fMRI voxel responses to predict the associated reversible capsule characteristics. The model presented in this chapter increases the accuracy of the structural similarity assessment measure by approximately $10 \%$ over the best current models, according to experimental validation using a publicly accessible handwritten digital image fMRI dataset. Furthermore, the proposed model's validity and generalizability are shown by displaying capsule characteristics and exposing the characterisation of certain voxels via gradient backpropagation. 

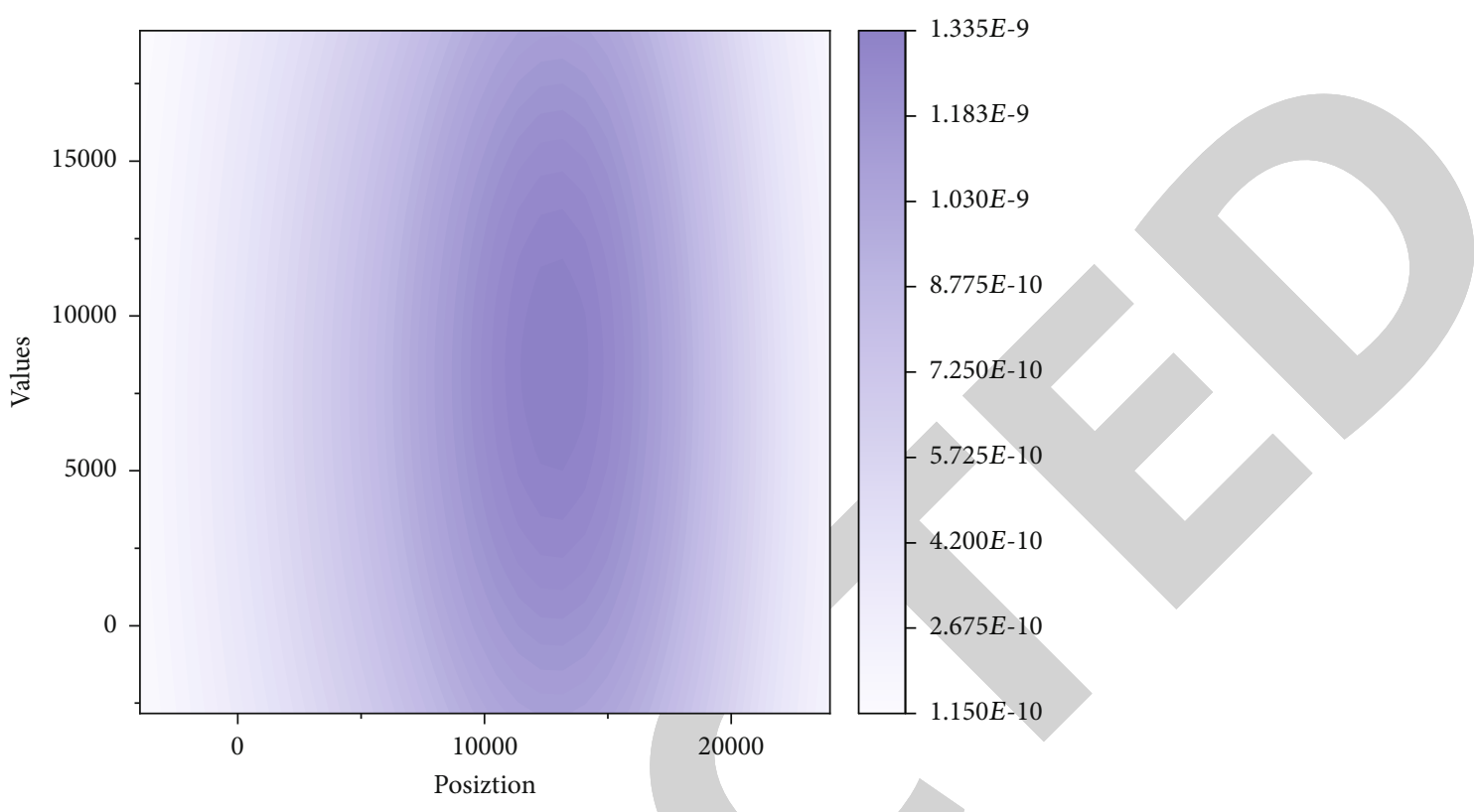

Figure 5: Voxel interpretation for feature visualization.

Since it is difficult to solve the visual reconstruction problem using the best visual coding model after completely solving the visual coding problem. Considering that the two opposite problems of visual coding and visual reconstruction can be mutually reinforcing, alternate training of encoder and decoder is designed. The initial encoder is trained without the aid of a decoder, so the initial encoder is first trained by minimizing the supervised loss function of the encoder. Following the training of the encoder, the decoder is trained by reducing decoder's semisupervised loss function. The encoder is retrained after receiving the trained decoder by reducing encoder's semisupervised loss function. The retrained encoder is more resilient than the originally trained encoder thanks to decoder training. Similarly, the decoder is retrained with a better encoder in order to enhance decoder reconfiguration. Thus, through alternating optimization, the encoder and decoder continually improve their encoding and decoding efficiency, resulting in a mutually reinforcing visual coding, and reconstruction paradigm shows the change of the loss function of two adjacent decoders (visual reconstruction) during the alternating optimization process, with different colors indicating different optimization orders. The red line represents the first optimization of the decoder, and the loss function gradually stabilizes and no longer decreases in the late stage of training, indicating that the current optimization has reached a bottleneck. Then, the encoder is trained, and after getting a better encoder, the decoder is trained again. The grey line represents the second optimization of the decoder, where the loss function of the decoder has a new drop again based on the first one. The loss function segments drop rapidly, as expected from the design of alternate training. The difference between the two optimizations is that the encoders used are different, and the second one is better compared to the first one, which brings an improvement in decoder performance. By cycling the alternating training, the encoder and decoder are continuously promoted to finally obtain a higher reconstruction performance (as shown in Figure 6).

Under the action of bottom-up and top-down visual mechanisms, different hierarchical visual areas are interconnected and correlated to characterise visual input information, whereas existing visual classification models feed all visual areas fMRI voxel responses into the visual classifier without exploiting the correlation between different visual areas, making it difficult to characterise the bottom-up and top-down information representation of visual cortex. Therefore, inspired by the bidirectional information flow in the visual cortex, decoding models are considered to design decoding models to characterize their inter-interior relationships using topological structure information between visual cortices to improve classification performance.

4.2. Results of the Experiment to Assess the Complexity of Chronic Pain in Aging Knee Osteoarthritis. The lateral tilt angle of trunk frontal plane: to investigate the effect of this index on the core index of this study, the data on the lateral tilt angle of the trunk during the complete support period in the gait cycle of three groups of subjects were extracted. To facilitate later data presentation and eigenvalue extraction, Figure 7 shows a graph of the change in trunk frontal plane internal tilt angle after the standardized support period.

This multiple linear regression is the result of fitting based on the original variables, the independent and dependent variables of the above equation are with physical units, and all variables are carrying real significance in their changes. The unstandardized regression coefficients reflect the absolute magnitude of the effect of changes in the independent variables on the dependent variable, while the standardized regression coefficients reflect the relative magnitude of the effect of different independent variables on the dependent variable, which can show the importance of the effect of different independent variables on the 


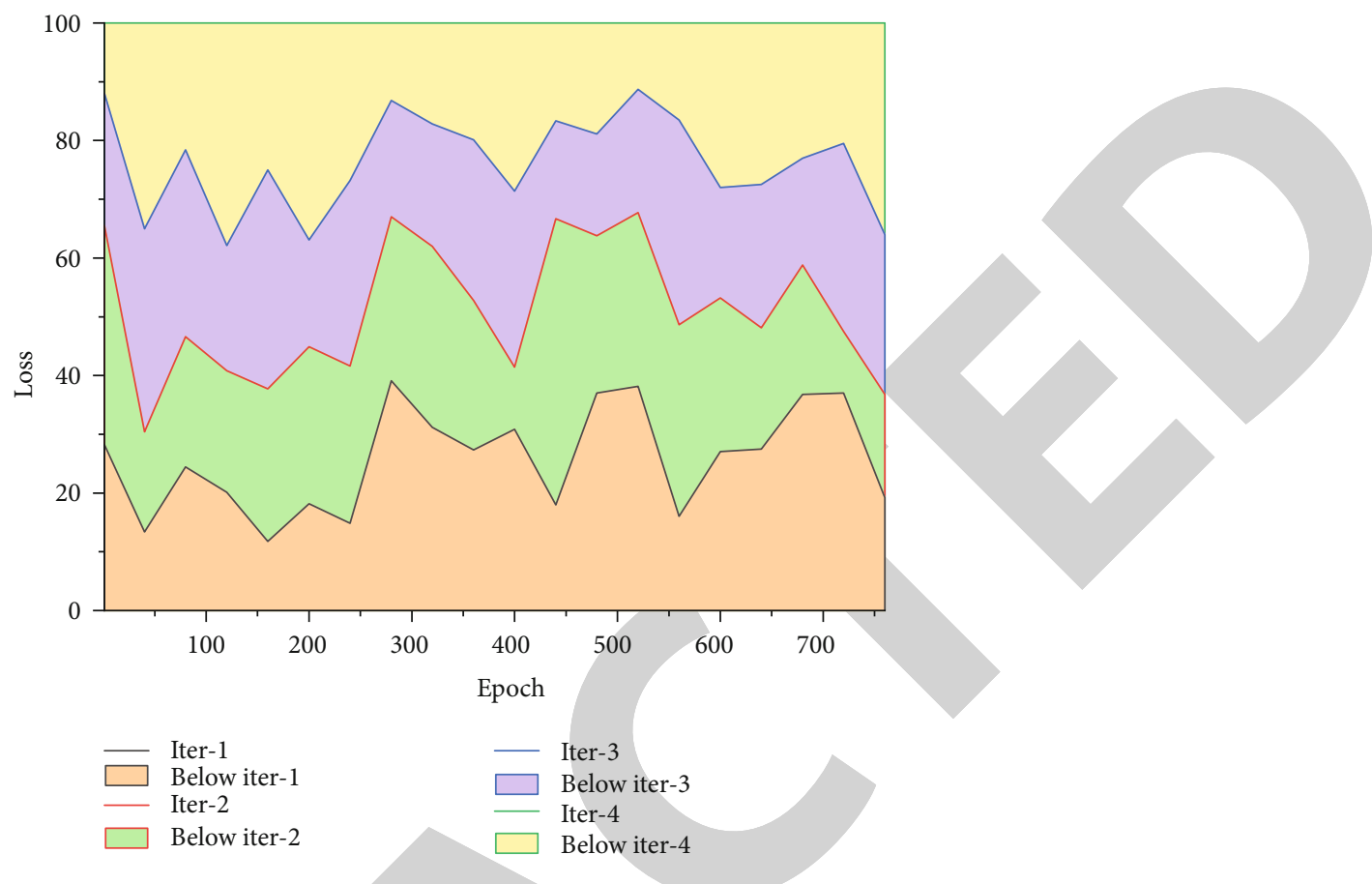

FIGURE 6: Change in decoder loss function during alternate training.

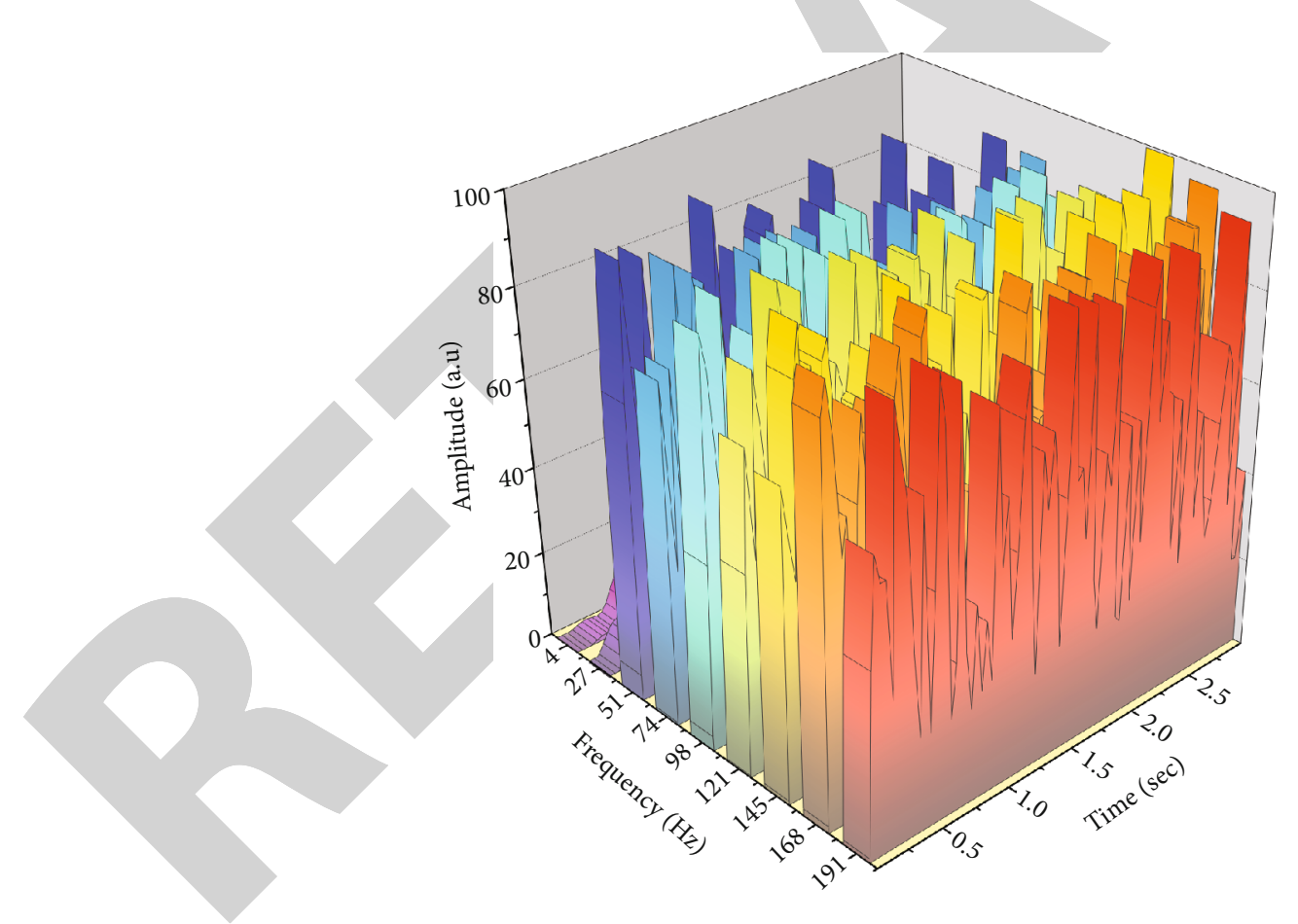

FIgURE 7: Curves of the angle of inclination within the frontal plane of the trunk during the period of gait support.

dependent variable. In this regression model, the standardized regression coefficients of several independent variables (BMI value/step speed/lateral trunk tilt angle/lateral pelvic tilt angle/foot advancement angle) were -0.521, 0.152, $0.088,-0.009$, and 0.140 . In order of absolute magnitude, the contributions were BMI value, step speed, foot advancement angle, lateral trunk tilt angle, and lateral pelvic tilt angle, in descending order. The contribution of BMI, gait speed, foot advancement angle, trunk lateral tilt angle, and pelvic lateral tilt angle was ranked in order of absolute value.

Studies have shown that neuroimaging markers of the brain predict pain perception as well as postsurgical analgesic effects. fMRI analysis may be more valuable than neuropsychological tests to detect cognitive decline in older KOA patients preparing for TKA treatment. Studies of diseasespecific measures of knee osteoarthritis have shown that 


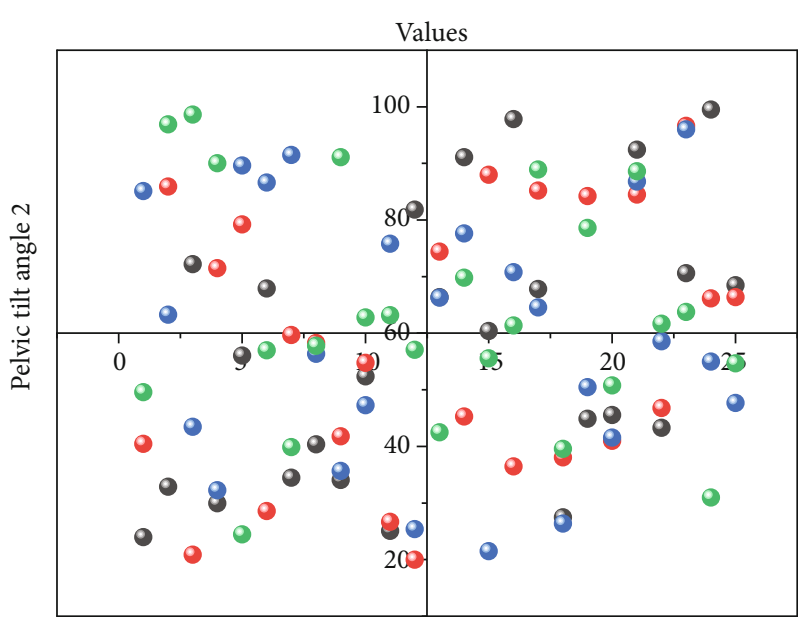

FIGURE 8: Estimation of the second peak curve of the independent variable and the knee joint inward moment.

the anterior insular cortex, prefrontal, and limbic areas are involved in the expected modulation of pain intensity. In female patients with severe KOA, aIC was associated with enhanced resting-state $\mathrm{FC}$ in the right orbitofrontal cortex (OFC), subpalpable areas, and bilateral frontal poles, and resting-state FC intensity in the left aIC and right OFC was positively correlated with KOA pain intensity. During painful stimulation, aIC is expected to activate OFC, subpalpable areas, frontal poles, and $\mathrm{aIC}$, and $\mathrm{FC}$ between brain regions influences severe KOA persistent postoperative pain formation, which can be combined with resting-state FC in preoperative specific pain areas to predict the degree of risk of persistent pain after TKA. Furthermore, in the context of neuroimaging studies of peripheral injurious stimuli, it has also been suggested that the analgesic effect of clinical procedures can be predicted by different types of coexisting MRI techniques combined with KOA pain with or without joint loading (as shown in Figure 8).

Similarly, recent studies have shown that knee inward moment magnitude is influenced by altered trunk motion in addition to significant hip abductor muscle weakness in KOA patients, which has been suggested as a risk factor for the development of knee osteoarthritis, and our results suggest that there is indeed lateralization of the pelvis during the support phase of walking, with some findings suggesting that lateral pelvic tilt may increase the magnitude of the internal knee moment; however, other research teams have suggested that hip abductor muscle strength has little effect on the internal hip and knee moments in gait, and the results of our subsequent regression analysis may explain the existence of this contradictory result. The concept of foot advancement angle has been mentioned previously, and by changing the angular bias of the long axis of the foot link, the position of the GRF action point in the foot can be influenced, thus changing the angle of the line connecting that point to the body's center of mass, which in turn influences the distance from the knee joint center to the GRF vector, and ultimately, the magnitude of the knee joint internal moment. Our findings corroborate this theoretical inference, with the two groups of subjects with greater BMI essentially maintaining a foot forward angle deviating from the forward direction and deflecting to the outside during the support phase of the walking process, commonly known as the external eight foot. Changes in ankle motion patterns also affect lower limb function, and the foot link forward angle is associated with the inward and outward knee moment in gait.

\section{Conclusion}

The use of neuroimaging methods in the research of pain processes in KOA allows for the assessment and prediction of central sensitization, emotional, and cognitive characteristics of the disease. Although the exact role of the relationship between disability, motor function, negative emotion, quality of life, and pain in KOA patients is unknown, recent neuroimaging studies have found that KOA patients' pain is closely related to the mediation of brain function and brain structure, laying the groundwork for future research. Currently, the diagnostic approach of correctly identifying pain components in KOA patients using neuroimaging methods has not been widely used in clinical practice, which may be due to the complexity of index processing and the high cost of neuroimaging examinations. Even while receiving the same KOA therapy, pain components in KOA patients with the same pain intensity may change with time, resulting in inconsistent analgesic effects. With technological advancements, the pain of KOA patients can be assessed using neuroimaging in the future, and a reasonable plan can be formulated to reduce the impact of different pain components on clinical efficacy as well as the potential side effects or medically derived injuries from clinical analgesic measures using neuroimaging technology. To summarise, neuroimaging technology has a bright future in the field of KOA pain diagnosis and treatment, and it will play a key role in individualised and precise treatment, as it can help to better identify treatment targets, generate new treatment ideas, and facilitate the development of reasonable protocols in the early and middle stages of KOA to improve clinical efficacy and safety.

\section{Data Availability}

The data used to support the findings of this study are included within the article.

\section{Conflicts of Interest}

The authors declare that they have no conflicts of interest.

\section{References}

[1] S. E. E. Mills, K. P. Nicolson, and B. H. Smith, "Chronic pain: a review of its epidemiology and associated factors in population-based studies," British Journal of Anaesthesia, vol. 123, no. 2, pp. e273-e283, 2019.

[2] D. J. Clauw, M. N. Essex, V. Pitman, and K. D. Jones, "Reframing chronic pain as a disease, not a symptom: rationale and implications for pain management," Postgraduate Medicine, vol. 131, no. 3, pp. 185-198, 2019. 
[3] E. Lluch, J. Nijs, C. A. Courtney et al., "Clinical descriptors for the recognition of central sensitization pain in patients with knee osteoarthritis," Disability and Rehabilitation, vol. 40, no. 23, pp. 2836-2845, 2018.

[4] T. Kurien and B. E. Scammell, "Mechanisms of pain in knee osteoarthritis that influence outcomes after total knee arthroplasty surgery," Bone \& Joint 360, vol. 10, no. 4, pp. 5-11, 2021.

[5] D. Borsook, J. Upadhyay, R. Hargreaves, and T. Wager, "Enhancing choice and outcomes for therapeutic trials in chronic pain: N-of-1 + imaging (+i)," Trends in Pharmacological Sciences, vol. 41, no. 2, pp. 85-98, 2020.

[6] K. S. Hemington, A. Rogachov, J. C. Cheng et al., "Patients with chronic pain exhibit a complex relationship triad between pain, resilience, and within- and cross-network functional connectivity of the default mode network," Pain, vol. 159, no. 8, pp. 1621-1630, 2018.

[7] A. Leroux, R. Rzasa-Lynn, C. Crainiceanu, and T. Sharma, "Wearable devices: current status and opportunities in pain assessment and management," Digital Biomarkers, vol. 5, no. 1, pp. 89-102, 2021.

[8] N. Vadivelu, A. M. Kai, G. Kodumudi, K. Babayan, M. Fontes, and M. M. Burg, "Pain and psychology—a reciprocal relationship," Ochsner Journal, vol. 17, no. 2, pp. 173-180, 2017.

[9] I. Tracey, C. J. Woolf, and N. A. Andrews, "Composite pain biomarker signatures for objective assessment and effective treatment," Neuron, vol. 101, no. 5, pp. 783-800, 2019.

[10] J. Liu, L. Chen, X. Chen et al., "Modulatory effects of different exercise modalities on the functional connectivity of the periaqueductal grey and ventral tegmental area in patients with knee osteoarthritis: a randomised multimodal magnetic resonance imaging study," British Journal of Anaesthesia, vol. 123, no. 4, pp. 506-518, 2019.

[11] S. Ravat, B. Olivier, N. Gillion, and F. Lewis PT, MSc, "Laterality judgment performance between people with chronic pain and pain-free individuals. A systematic review and meta-analysis," Physiotherapy Theory and Practice, vol. 36, no. 12, pp. 1279-1299, 2020.

[12] M. P. Klinck, J. S. Mogil, M. Moreau et al., "Translational pain assessment: could natural animal models be the missing link?," Pain, vol. 158, no. 9, pp. 1633-1646, 2017.

[13] J. Mathew, D. B. Adhia, M. L. Smith, D. de Ridder, and R. Mani, "Protocol for a pilot randomized sham-controlled clinical trial evaluating the feasibility, safety, and acceptability of infraslow electroencephalography neurofeedback training on experimental and clinical pain outcomes in people with chronic painful knee," NeuroRegulation, vol. 7, no. 1, pp. 3044, 2020.

[14] M. A. Ruiz Iban, J. Benavides, J. P. Forero et al., "Use of strong opioids for chronic pain in osteoarthritis: an insight into the Latin American reality," Expert Review of Clinical Pharmacology, vol. 11, no. 1, pp. 47-59, 2018.

[15] S. P. Cohen, L. Vase, and W. M. Hooten, "Chronic pain: an update on burden, best practices, and new advances," The Lancet, vol. 397, no. 10289, pp. 2082-2097, 2021.

[16] H. Huang, J. Tanner, H. Parvataneni et al., "Impact of total knee arthroplasty with general anesthesia on brain networks: cognitive efficiency and ventricular volume predict functional connectivity decline in older adults," Journal of Alzheimer's Disease, vol. 62, no. 1, pp. 319-333, 2018.

[17] H. Nahman-Averbuch, V. J. Schneider, L. A. Chamberlin et al., "Identification of neural and psychophysical predictors of headache reduction after cognitive behavioral therapy in adolescents with migraine," Pain, vol. 162, no. 2, pp. 372-381, 2021.

[18] Y. Zhao and M. Talha, "Evaluation of food safety problems based on the fuzzy comprehensive analysis method," Food Science and Technology, 2021.

[19] J. Y. Ro, Y. Zhang, C. Tricou, D. Yang, J. T. da Silva, and R. Zhang, "Age and sex differences in acute and osteoarthritis-like pain responses in rats," The Journals of Gerontology: Series A, vol. 75, no. 8, pp. 1465-1472, 2020.

[20] B. McCarberg and J. Peppin, "Pain pathways and nervous system plasticity: learning and memory in pain," Pain Medicine, vol. 20, no. 12, pp. 2421-2437, 2019.

[21] H. Si, C. Wang, Y. Jin et al., "Prevalence, factors, and health impacts of chronic pain among community- dwelling older adults in China," Pain Management Nursing, vol. 20, no. 4, pp. 365-372, 2019. 